\title{
ASYMPTOMATIC EXTRUSION OF ANTERIOR CERVICAL SPINE IMPLANT FROM HYPOPHARYNX: A CASE REPORT
}

\author{
(1) Nilesh BARWAR, (1) Bharat SHARMA
}

All India Institute of Medical Sciences, Department of Orthopaedics, Jodhpur, India

Anterior cervical spine surgery for cervical disc radiculopathy was first described by Smith and Robinson. Hardware placement in the anterior cervical spine began in the 1980s, primarily for anterior stabilisation of cervical spine trauma. Later, its use extended into the management of cervical radiculopathy in the form of discectomy and arthrodesis. Anterior cervical discectomy and fusion and anterior cervical corpectomy and fusion are now well-recognised as favourable methods of fixation for cervical spine spondylotic myelopathy, traumatic spine, ossification of posterior longitudinal ligament, neoplasia and infection. However, numerous complications have been reported following anterior cervical surgery. Immediate complications include oesophageal rupture, recurrent laryngeal nerve injury, cerebrospinal fluid leakage, vascular complications, worsening of neurological status, etc. Among late complications, they are systemic sepsis, abscess formation, mediastinitis, screw pullout, plate failure and fistula formation, etc. We present a case report of a 64-year-old male who presented with spontaneous asymptomatic dislodgement of parts of the anterior cervical plating system through the posterior wall of hypopharynx 2 years after the index surgery.

Keywords: Asymptomatic, extrusion, cervical implant

\section{INTRODUCTION}

Anterior cervical spine procedures with instrumented fusion are the procedures of choice for many cervical spine pathologies. Dislodgement of hardware is a known complication in the literature. Diagnosis of dislodgement of hardware following anterior cervical discectomy and fusion (ACDF) and anterior cervical corpectomy and fusion (ACCF) is difficult because dysphagia is common, with a prevalence ranging from $2 \%$ to $60 \%{ }^{(1)}$. Around $14 \%$ of patients have reported persistent dysphagia up to 2 years after ACDF surgery ${ }^{(2)}$. However, in most patients, dysphagia resolves spontaneously without any intervention. Only $2 \%$ require further intervention ${ }^{(3)}$. Most commonly, the pulled out screw extrudes from esophagus ${ }^{(4)}$. We present a case of asymptomatic screw extrusion from the hypopharynx, which is quite rare.

\section{CASE REPORT}

A 64-year-old male presented with progressive weakness and numbness in upper and lower limbs for the last one year. He was unable to do occupational writing work and his daily routine activities. There was no involvement of bowel and bladder. After detailed investigations, he was diagnosed with cervical spine spondylotic myelopathy (Figure 1). He underwent C4 corpectomy, decompression of spinal cord and fixation from C3 to C5 using an anterior plating system and titanium mesh cage with autologous bone graft (Figure 2). Surgery was uneventful and patient got improved by 2 weeks after surgery. Three months after the surgery, the patient was again able to do occupational writing work as well as his daily routine activities. The patient was asymptomatic until 2 years after the index surgery, when one day he developed a feeling of a foreign body in his throat, which caused forceful cough reflex. A screw was expelled out from his mouth. Due to a busy festival season and asymptomatic presentation, the patient did not rush to the hospital. After a week, he presented with the dislodged screw in his hand. Plain radiograph confirmed a missing screw from the plate (Figure 3). Direct laryngoscopy and endoscopy were done to see if there was a fistula or abscess in the pharynx or oesophagus. We found a blood clot with the fistula underneath (Figure 4). Computed tomography (CT) scan confirmed a fistula track from cervical spine to hypopharynx (Figure 5). Though the fistula was present, as it was visualised by non-contrast CT scan and laryngoscopy, the patient did not have any complaint in his throat until the last follow-up. Therefore, we decided to keep him under observation. 


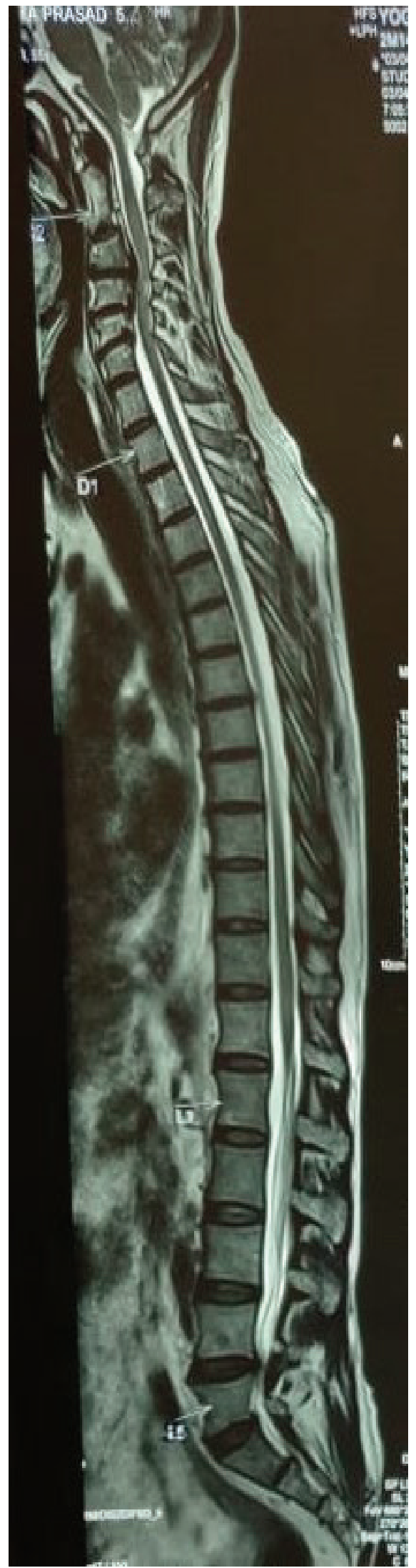

Figure 1. MRI T2 sag of C-spine with CSM

sag: Sagittal, CSM: Cervical spondylotic myelopathy, MRI: Magnetic resonance imaging

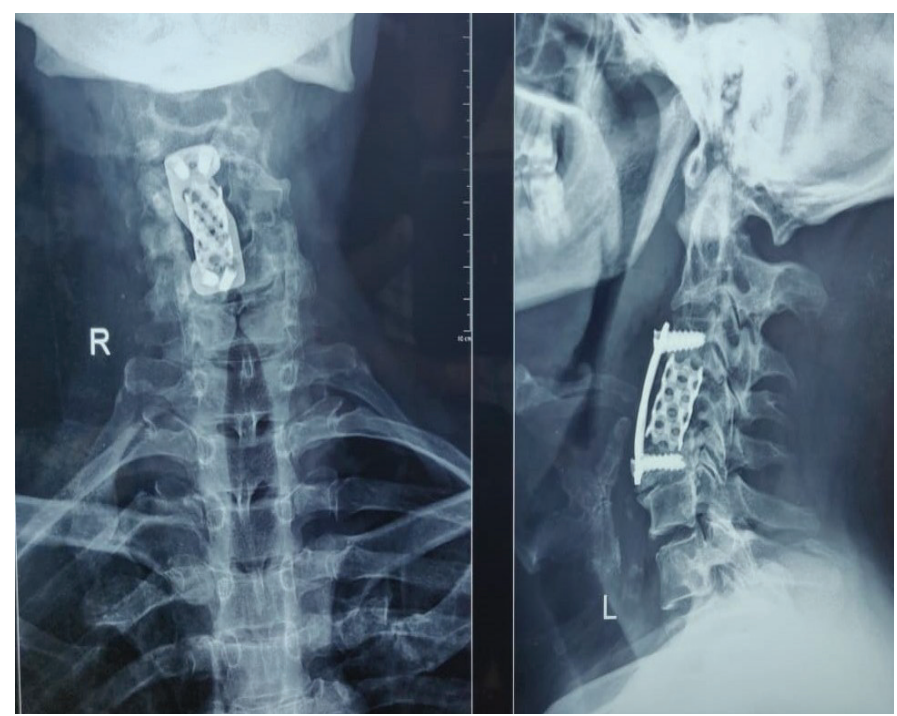

Figure 2. Post-operative X-ray with anterior plate and Harm's cage at $\mathrm{C4}$

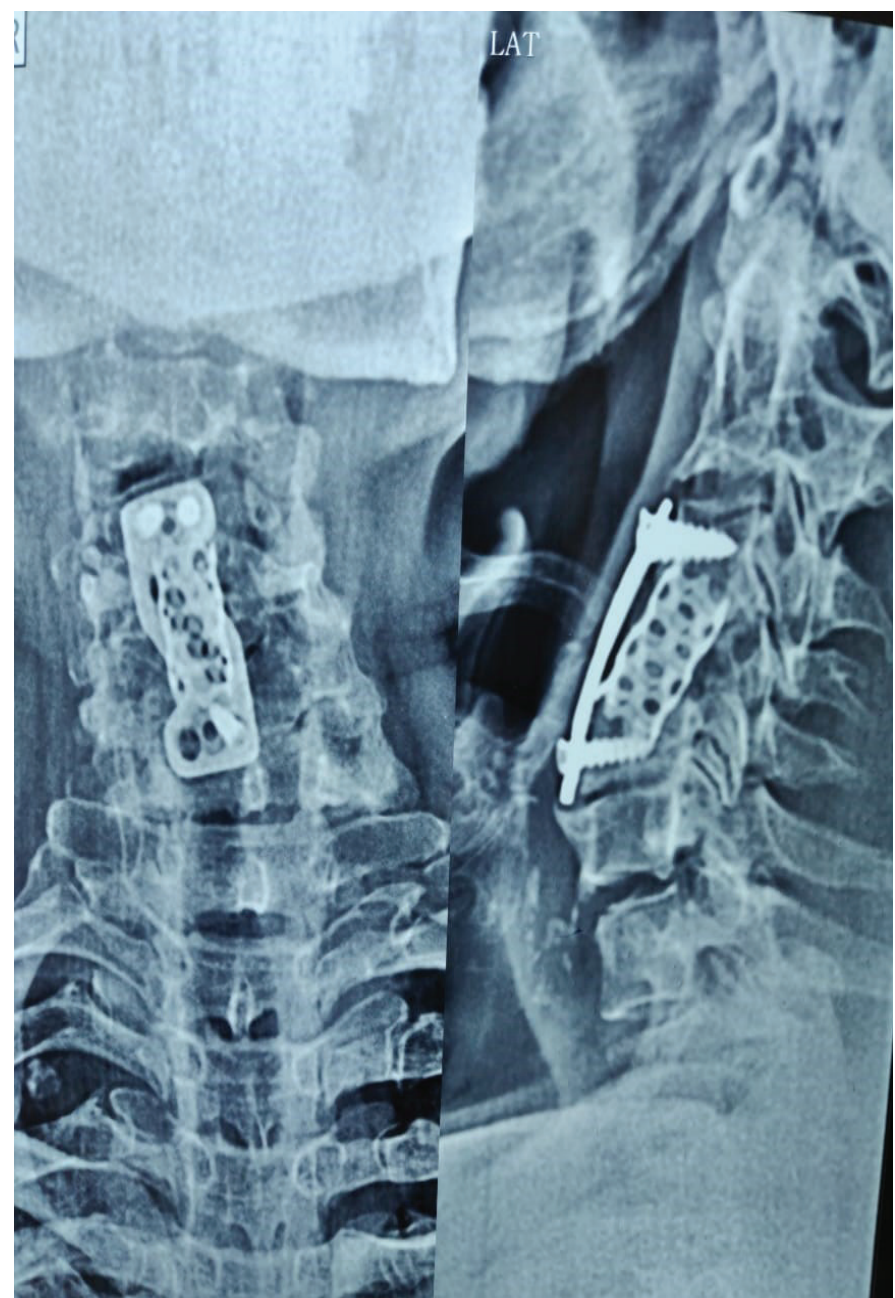

Figure 3. X-ray C-spine AP \& Lat view showing missing screw AP: Anteroposterior, Lat: Lateral 


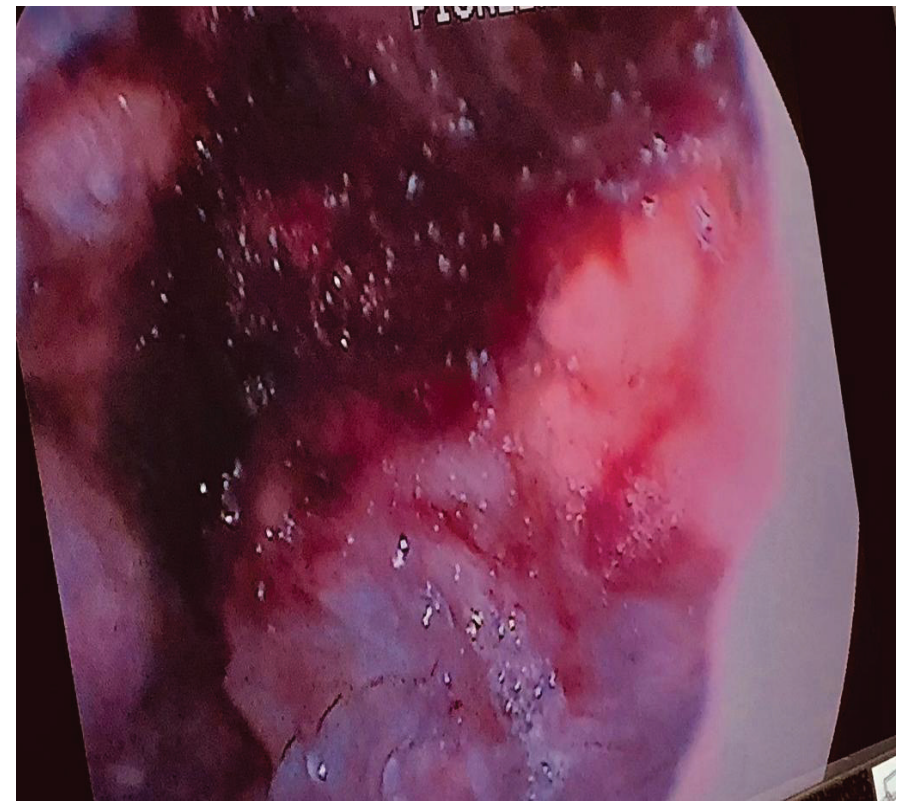

Figure 4. Laryngoscopy showing blood clot on posterior wall of hypopharynx with fistula underneath

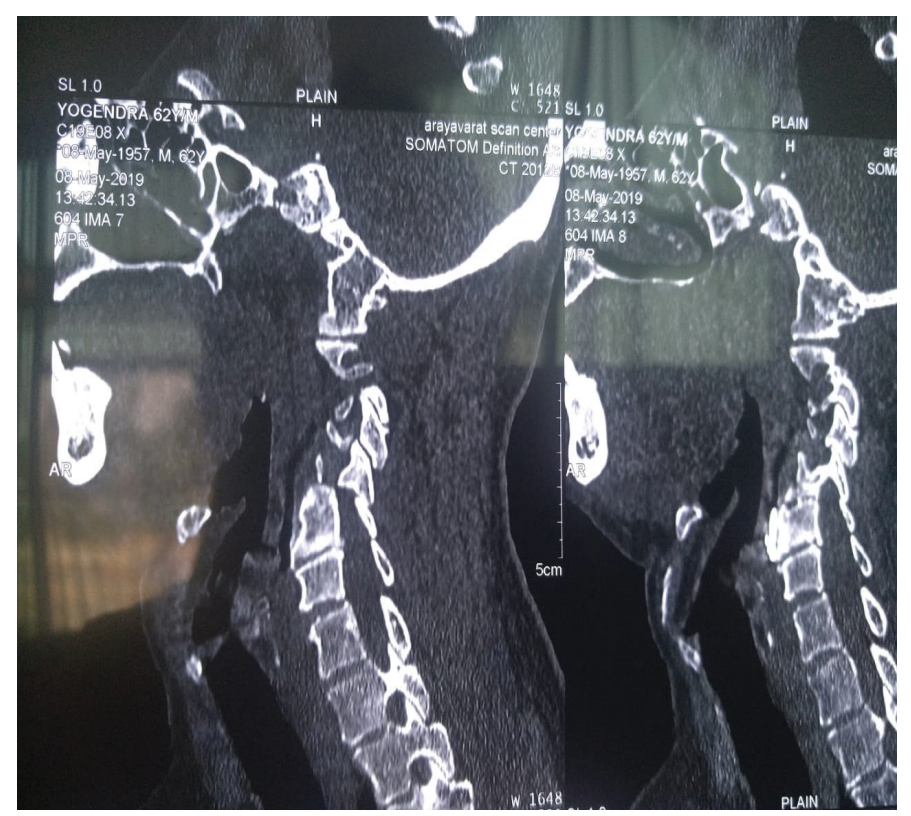

Figure 5. CT scan of C-spine, confirming fistula track communicating to hypopharynx

$\mathrm{CT}$ : Computed tomography

\section{DISCUSSION}

ACDF/ACCF procedures are widely used for many pathologic cervical conditions due to advanced improvement in hardware designs and widespread familiarity of surgeons with anterior cervical spine approach(3). Similar to any other surgery, anterior cervical spine procedures with hardware placement are not free from complications. Many early complications may resolve with simple conservative management, but late complications generally need surgical intervention(5). Ning et al.(1) found 239 complications in 2,233 patients, including screw loosening in 37 (1.7\%) patients, plate loosening in 72 (3.2\%) patients, broken screw in four $(0.2 \%)$ patients and broken plate in two $(0.1 \%)$ patients. Lowery and McDonough ${ }^{(6)}$ found hardware failure in 38 (35\%) patients out of 109 . Sun et al. ${ }^{(7)}$ observed oesophageal fistulas in five patients out of 2,348 patients. Similarly, Tasiou et al. ${ }^{(5)}$ found in one $(0.9 \%)$ patient with implant dislodgement. Korovessis et al. ${ }^{(8)}$ reported oesophageal perforation associated with spondylodiscitis. In addition, early life-threatening complications are also reported. Li et al.(9) presented a case report of acute cervical hematoma, screw pull-out and oesophageal perforation. Sometimes, late complications of ACDF are life-threatening and should be addressed aggressively. Wong et al. ${ }^{(10)}$ reported a case of acute airway obstruction by pre-vertebral abscess formation and a missing screw. Screw expulsion from oesophagus and fistula formation is welldocumented in the literature but asymptomatic dislodgement from the hypopharynx is very rare presentation.

With use of a third-generation locking plate system, implant failure rate is reduced; however, higher cervical fixation gives small surgical field for optimum screw position. Two cortex purchases were a strict requisite for old non-locking plate system, but unicortical purchase is sufficient in locking plate system. Ours was a non-locking screw plate system.

Every patient with a complaint of dysphagia should be evaluated in detail to avoid life- threatening complications. Patients presenting with an extruded screw should be considered as having a fistula unless proven otherwise. Work-up should include complete blood count, erythrocyte sedimentation rate, C-reactive protein, plain radiographs, $\mathrm{CT}$ scan, magnetic resonance imaging, laryngoscopy and endoscopy. Vital stability, nasogastric tube placement, nutritional and fluid maintenance intravenous broad-spectrum antibiotics and urgent surgical intervention are the mainstay treatment. Entangled implants may have to be removed urgently depending on location. A fistula can be repaired with simple suturing to a muscle flap,such as the pectoralis or sternocleidomastoid flap, in collaboration with an ear, nose and throat surgeon. Preoperative evaluation of bone quality and systemic diseases, careful scrutiny for anatomical anomalies, use of improved new generation plating system and appropriate surgical techniques may decrease the chances of screw dislodgement following instrumented anterior cervical spine fusion. We advise not underestimating a complaint of dysphagia or foreign body sensation in a patient who has previously undergone ACDF/ACCF surgery. Every patient must undergo laryngoscopy and endoscopy with plain radiographs of the spine and abdomen to rule out any dislodgement of the implant.

In conclusion, our case report is a rare presentation. Until now, oesophageal screw extrusion with fistula and abscess formation is well-documented in the literature. Immediate complications like oesophageal rupture, hematoma formation, cerebrospinal fluid leakage and very late complications like infection, plate 
migration and hardware failure were recognised well in the past, but asymptomatic presentation from hypopharynx in this interval of time after surgery is still rare. Gradual or acute onset dysphagia with or without dyspnoea in a patient with anterior cervical spine surgery with implant warrants an urgent search for dislodged implant and its appropriate management.

\section{Ethics}

Informed Consent: Consent was taken from the patient himself. Peer-review: Externally and Internally peer-reviewed.

\section{Authorship Contributions}

Concept: N.B., Design: B.S., Data Collection or Processing: B.S., Analysis or Interpretation: N.B., Literature Search: N.B., Writing: N.B., B.S.

Conflict of Interest: No conflict of interest was declared by the authors.

Financial Disclosure: The authors declared that this study received no financial support.

\section{REFERENCES}

1. Ning $X$, Wen $Y$, Xiao-Jian $Y, B i n ~ N$, De-Yu C, Jian-Ru X, et al. Anterior cervical locking plate-related complications; prevention and treatment recommendations. Int Orthop. 2007;32:649-55.

2. Lee MJ, Bazaz R, Furey CG, Yoo J. Risk Factors for Dysphagia After Anterior Cervical Spine Surgery: A Two-Year Prospective Cohort Study. Spine J. 2007;7:141-7.
3. Nathani A, Weber AE, Wahlquist TC, Graziano GP, Park P, et al. Delayed presentation of pharyngeal erosion after anterior cervical discectomy and fusion. Case Rep Orthop. 2015, doi: 10.1155/2015/173687.

4. Salis G, Pittore B, Balata G, Bozzo C. A rare case of hypopharyngeal screw migration after spine stabilization with plating. Case Rep Otolaryngol. 2013, doi: 10.1155/2013/475285.

5. Tasiou A, Giannis T, Brotis AG, Siasios I, Georgiadis I, Gatos H, et al. Anterior cervical spine surgery-associated complications in a retrospective case-control study. J Spine Surg. 2017;3:444-59.

6. Lowery GL, McDonough RF. The significance of hardware failure in anterior cervical plate fixation. Patients with 2- to 7-year follow-up. Spine (Phila Pa 1976). 1998;23:181-6.

7. Sun L, Song YM, Liu LM, Gong Q, Liu H, Li T, et al. Causes, treatment and prevention of esophageal fistulas in anterior cervical spine surgery. Orthop Surg. 2012;4:241-6.

8. Korovessis P, Repantis T, Vitsas V, Vardakastanis K. Cervical spondylodiscitis associated with oesophageal perforation: a rare complication after anterior cervical fusion. Eur J Orthop Surg Traumatol. 2013;23(suppl 2):S159-63.

9. Li Y, Zhu QS, Liu JC, Wu YT. Acute cervical epidural hematoma, screw pullout, and esophageal perforation after anterior cervical corpectomy surgery: report of a case. Int Surg. 2015;100:334-40.

10. Wong DT, Fehlings MG, Massicotte EM. Anterior cervical screw extrusion leading to acute upper airway obstruction: case report. Spine (Phila Pa 1976). 2005;30:E683-6. 